\title{
Emission and Absorption Tuning in MR-TADF B,N-Doped Hep- tacenes: Towards Ideal-Blue Hyperfluorescent OLEDs
}

\author{
Kleitos Stavrou, ${ }^{a t}$ Subeesh Madayanad Suresh, ${ }^{b *}$ David Hall, ${ }^{b, d}$ Andrew Danos, ${ }^{a *}$ \\ Nadzeya A. Kukhta, ${ }^{a+}$ Alexandra M. Z. Slawin, ${ }^{b}$ Stuart Warriner, ${ }^{c}$ David Beljonne, ${ }^{d}$ \\ Yoann Olivier, ${ }^{\text {dee }}$ Andrew Monkman, ${ }^{a}$ and Eli Zysman-Colman ${ }^{b *}$ \\ ${ }^{a}$ Department of Physics, Durham University, Durham, UK, DH1 3LE. \\ E-mail: andrew.danos@durham.ac.uk
}

\begin{abstract}
${ }^{b}$ Organic Semiconductor Centre, EaStCHEM School of Chemistry, University of St Andrews, St Andrews, UK, KY16 9ST. E-mail: eli.zysman-colman@st-andrews.ac.uk
\end{abstract}

${ }^{c}$ School of Chemistry, University of Leeds, Woodhouse Lane, Leeds, UK

${ }^{d}$ Laboratory for Chemistry of Novel Materials, University of Mons, 7000, Mons, Belgium.

${ }^{e}$ Laboratory for Computational Modeling of Functional Materials, Namur Institute of Structured Matter, Université de Namur, Rue de Bruxelles, 61, 5000 Namur, Belgium.

\$ These authors contributed equally

+ Current address: Materials Science and Engineering Department, University of Washington, Seattle, Washington 98195-2120, United States.

\begin{abstract}
Developing high-efficiency purely organic blue organic light-emitting diodes (OLEDs) that meet the stringent industry standards is a major current research challenge. Hyperfluorescent device approaches achieve in large measure the desired high performance by combining the advantages of a high-efficiency thermally activated delayed fluorescence (TADF) assistant dopant with a narrowband deep-blue multi-resonant TADF (MR-TADF) terminal emitter. However, this approach requires suitable spectral overlap to support Förster resonance energy transfer (FRET) between the two.
\end{abstract}

Here we demonstrate colour tuning of a recently reported MR-TADF B,N-heptacene core through control of the boron substituents. While there is little impact on the intrinsic TADF properties - as both 
singlet and triplet energies decrease in tandem - this approach improves the emission colour coordinate as well as the spectral overlap for blue hyperfluorescence OLEDs (HF OLEDs). Crucially, the redshifted and more intense absorption allows us to pair this MR-TADF emitter with a high-performance TADF assistant dopant and achieve maximum external quantum efficiency (EQE $\mathrm{E}_{\max }$ ) of $15 \%$ at colour coordinates of $(0.15,0.10)$. The efficiency values recorded for our device at a practical luminance of $100 \mathrm{~cd} \mathrm{~m}^{-2}$ are among the highest reported for HF TADF OLEDs with $\mathrm{CIE}_{\mathrm{y}} \leq 0.1$.

\section{Introduction}

Organic light-emitting diode (OLED) display technology is evolving at a brisk rate, with high-end ultrahigh definition (UHD) 4K and 8K OLED displays already in the market. These high-resolution displays must meet stringent emission colour standards (BT.2020-2), ${ }^{1}$ which are defined according to the Commission International de l'Éclairage (CIE) 1931 as $(0.13,0.05),(0.17,0.80)$ and $(0.71,0.29)$ for blue, green, and red, respectively. ${ }^{2}$ At present this colour requirement is met through the use of absorptive filters or microcavities to deliver saturated blue, green, and red emission. ${ }^{3,4}$ Unfortunately this approach necessarily results in a reduced efficiency of the devices as unwanted emission wavelengths are rejected. An alternative and attractive solution to the issue of colour purity is to develop materials with intrinsically narrowband emission.

Commercial OLED displays currently use organic triplet-triplet annihilation (TTA) emitters for blue pixels. By virtue of the operational exciton harvesting mechanism, the internal quantum efficiency of blue OLEDs is presently capped at $62.5 \%$. Organic thermally activated delayed fluorescence (TADF) materials, by contrast, can harvest $100 \%$ of the electrically generated excitons to produce light and achieve higher device efficiencies. ${ }^{5}$ Similarly, organometallic phosphorescent OLEDs can generate high-efficiency OLEDs, although with significant intrinsic limitations for blue-emitting device in terms of their stability. These limitations arise as photon energies approach metal-ligand bond dissociation energies, and as thermal population of metal-centred states (particularly for $\mathrm{d}_{6}$ metal complexes) leads to severe non-radiative decay.

The TADF mechanism involves the thermal upconversion of non-emissive triplet excitons into singlets via reverse intersystem crossing (RISC). A small energy gap between $\mathrm{T}_{1}$ and $\mathrm{S}_{1}\left(\Delta E_{\mathrm{ST}}\right)$ is necessary to 
achieve TADF, and this is ensured when there is spatial separation of the frontier orbitals, which is normally achieved in a highly twisted donor-acceptor (D-A) architecture. However, D-A TADF emitters typically emit from charge transfer (CT) states where there is a large reorganization in the excited state that leads to broad (70-100 nm) emission. ${ }^{6,7}$ These broad emission bands interact poorly with the aforementioned colour purity filters, while also making it difficult to engineer deep-blue emitters ${ }^{8-10}$ and suitably high-triplet hosts. ${ }^{11}$

Multiresonant TADF (MR-TADF) compounds are an alternative class of TADF materials that are typically based on p- and n-doped nanographene (Figure S1). ${ }^{12,13}$ Because of their rigid structure, these compounds show narrowband emission, typically with full width of half maxima (FWHM) of around 20-30 nm. Thus, MR-TADF emitters show potential to generate the required deep blue emission demanded by industry, and support the development of stable, efficient, pure blue narrowband emitting organic materials that are expected to revolutionize OLED displays. ${ }^{14}$ MR-TADF compounds show TADF due to the alternating pattern of electron density between the ground and excited states that leads to small $\Delta E_{\mathrm{ST}}$, combined with upper-triplet crossings from thermally-populated $\mathrm{T}_{\mathrm{n}}$ states back to the emissive $\mathrm{S}_{1}$ state. $^{15-18}$ The excited states thus possess a distinct short range charge transfer (SRCT) character, ${ }^{13,19,20}$ and MR-TADF compounds are endowed with high singlet radiative decay $\left(k_{\mathrm{r}}\right)$ rates of around $10^{7} \mathrm{~s}^{-1} \cdot 5,19$

Despite these advantages, the RISC rates reported for MR-TADF materials typically lag $\sim 100$ times slower than those of leading D-A or D-A-D TADF materials. ${ }^{13,21}$ This limitation frequently leads to inefficient triplet harvesting and low performance of the OLEDs at practical luminance. To circumvent this limitation, MR-TADFs have recently found a parallel application as terminal emitters in hyperfluorescent (HF) OLEDs. Indeed, truly ground-breaking device performances have been achieved when pairing a high-RISC D-A assistant dopant with narrowband MR-TADF terminal emitters. ${ }^{22-24}$ This performance is in some cases supported by spontaneous alignment of the MR-TADF with the substrate, thus improving optical outcoupling of the device, ${ }^{24}$ although the specific loss mechanisms that occur under electrical excitation, including charge trapping and Dexter transfer, ${ }^{25}$ remain poorly understood. 
High-performance HF OLEDs rely on efficient energy transfer between the D-A TADF assistant dopant that is responsible for exciton harvesting and the MR-TADF terminal emitter. Förster resonance energy transfer (FRET) is understood to be the main mechanism for this transfer, and FRET efficiency is proportional to the overlap between the emission spectrum of the D-A TADF assistant dopant and the absorption spectrum of the MR-TADF terminal emitter. As a result, the compatibility of many MRTADF materials is severely limited by the low molar extinction coefficient of their lowest energy absorption bands - those that predominately overlap with the emission spectrum of the D-A TADF assistant dopant. Expanding the FRET compatibility of a specific terminal emitter requires increasing the spectral overlap between the assistant dopant and terminal emitter. This in turn requires development of deeper-blue D-A TADF assistant dopants, but such emitters remain persistently elusive - a circumstance that has stimulated the rapid development of the alternate hyperfluorescence approach.${ }^{26}$ Clearly then, synthetic control over both the emission and absorption spectra ${ }^{27-29}$ of MR-TADF materials is crucial to enable and optimise their use in HF OLEDs with available D-A TADF co-hosts.<smiles>Cc1ccc(N2c3ccc(C)cc3B(O)c3cc4c(cc32)B(O)c2cc3c(cc2N4c2ccc(C)cc2)B(O)c2cc(C)ccc2N3c2ccc(C)cc2)cc1</smiles>

$\alpha-3 B N O H$

J. Am. Chem. Soc. 2020, 142, 6588-6599

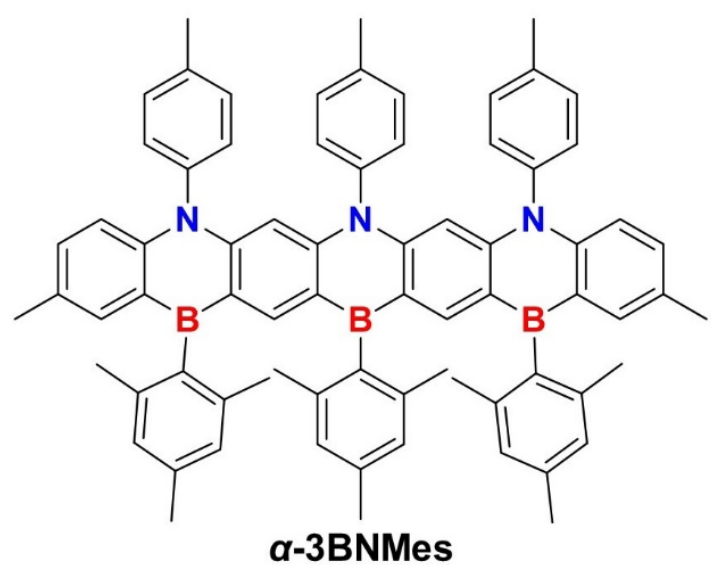

This work

Figure 1. Structures of $\alpha-3 B N O H$ and $\alpha-3 B N M e s$.

We previously reported a linear B,N-doped ladder type heptacene that emits at $390 \mathrm{~nm}$ (near UV) with a FWHM of $31 \mathrm{~nm}(240 \mathrm{meV})$ in THF solution. ${ }^{30}$ The material displayed weak TADF due in part to the large $\Delta E_{\mathrm{ST}}$ of $0.31 \mathrm{eV}$, along with significant TTA contribution reflected in the extended ms-timescale of the emission decay. ${ }^{30}$ Due to the near UV emission and subsequent paucity of suitable OLED hosts, the investigated device performance was poor. ${ }^{31}$ In the present report we show how replacement of the 
hydroxyl groups for mesityl substituents in $\boldsymbol{\alpha - 3 B N M e s ~ ( F i g u r e ~ 1 ) ~ l e a d s ~ t o ~ a ~ d e s i r e d ~ r e d - s h i f t i n g ~ o f ~ t h e ~}$ emission towards an ideal-blue emission colour coordinate, as well as a red-shifting of the absorption that supports HF compatibility with available D-A TADF assistant dopants. Together, these changes in optical properties compared to $\boldsymbol{\alpha}$-3BNOH allow $\boldsymbol{\alpha}$-3BNMes to be used in high-performance deep-blue HF OLEDs that show $15 \%$ maximum external quantum efficiencies $\left(\mathrm{EQE}_{\max }\right)$ and colour coordinates of $(0.15,0.10)$. These results represent highly competitive device performance metrics at this colour coordinate that are enabled by the finely-tuned emission and absorption spectra of $\boldsymbol{\alpha}$-3BNMes (Table S2).

\section{Results and Discussion}

\section{Synthesis}

$\boldsymbol{\alpha}$-3BNMes was synthesized in three steps (Scheme S1), where the key borylation step proceeds in 57\% yield. The identity and purity of $\boldsymbol{\alpha - 3 B N M e s}$ were established from a combination of ${ }^{1} \mathrm{H}$ NMR spectroscopy (S2), high-resolution mass spectrometry (S4), HPLC (S5), GPC trace analyses (S7) and single crystal X-ray diffraction analysis (Figure 2). Like the parent compound ( $\boldsymbol{\alpha}-\mathbf{3 B N O H}), \boldsymbol{\alpha}-\mathbf{3 B N M}$ Mes shows high thermal stability, revealed by thermogravimetric analysis (TGA), with a decomposition temperature $\left(\mathrm{T}_{\mathrm{d}}\right)$, defined as the $5 \%$ weight loss of the material, at $503{ }^{\circ} \mathrm{C}$ (Figure S8).

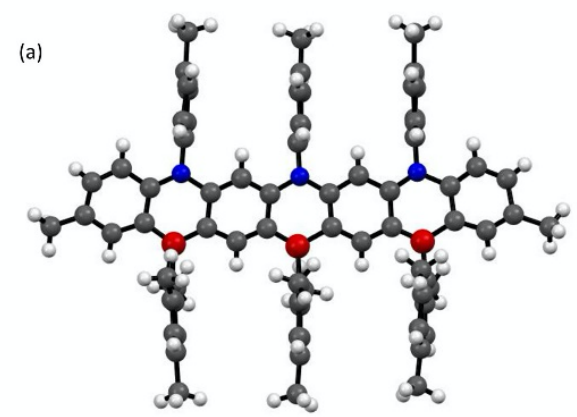

(b)

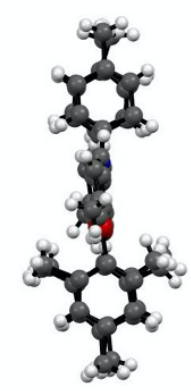

(c)

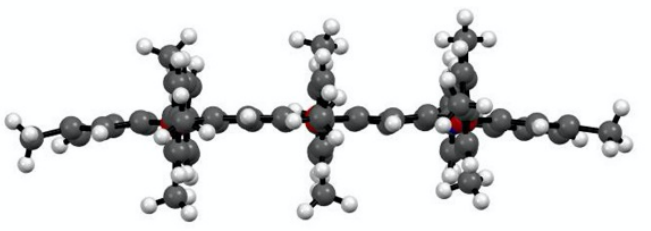

Figure 2. (a) ball and stick representation of $\boldsymbol{\alpha}$-3BNMes obtained by single crystal X-ray analysis. (b) side and (c) plane views. 
We investigated the structure of $\boldsymbol{\alpha - 3 B N M e s}$ by growing single crystals via slow evaporation of the compound in THF. Full datasets were collected from many different crystals, and we report here our best result. The data obtained are adequate to demonstrate connectivity and gross structure but do not merit discussion of bond lengths (Figure 2). Like the parent $\boldsymbol{\alpha}-\mathbf{3 B N O H}$, in $\boldsymbol{\alpha}-\mathbf{3 B N M e s}$ the heptacene core remains planar, and the aryl substituents attached to boron and nitrogen align nearly orthogonal to main acene core.

(a)

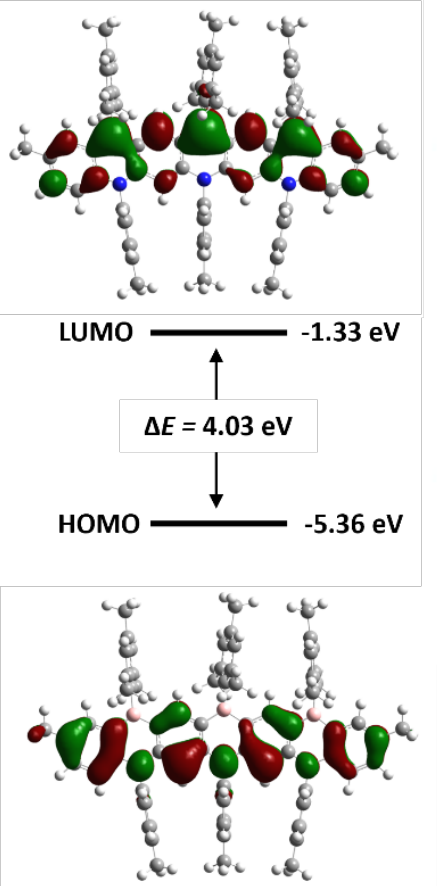

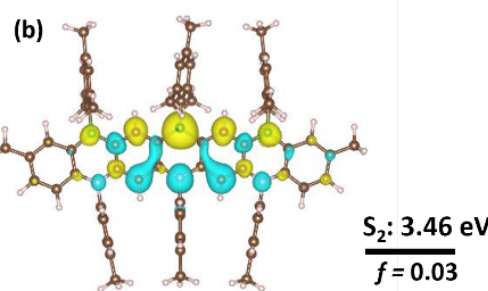
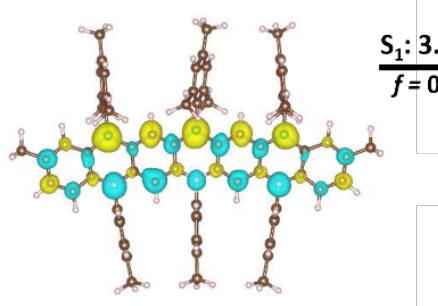

$\frac{\mathrm{S}_{1}: 3.40 \mathrm{eV}}{f=0.08}$ $\mathrm{T}_{2}: 3.22 \mathrm{eV}$ $\Delta E_{\mathrm{sT}}: 0.20 \mathrm{eV}$

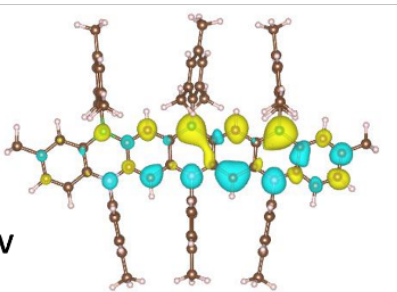

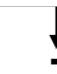
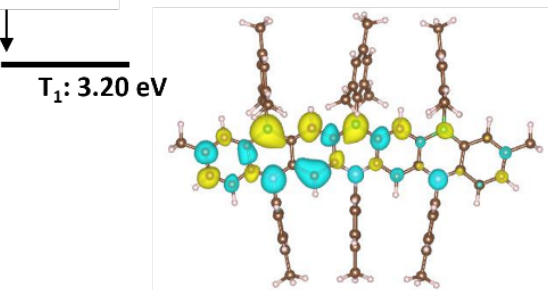

Figure 3. a) HOMO (bottom) and LUMO (top) energy distribution in $\boldsymbol{\alpha}$-3BNMes calculated using PBE0/6-31G $(\mathrm{d}, \mathrm{p})$, isovalue $=0.02$; (b) Difference density plots for the lowest-lying two singlets and triplets excited states for $\boldsymbol{\alpha}$-3BNMes calculated in the gas phase by using SCS-CC2/cc-pVDZ. Blue colour represents an area of decreased electron density and yellow represents an increased electron density between the ground and excited states, isovalue $=0.001$.

Computational modelling provides a clear description of this MR-TADF compound. DFT calculations at the PBE0/6-31G(d,p) level of theory in the gas phase predict a large HOMO-LUMO gap of $4.03 \mathrm{eV}$. The electron density distribution patterns of these orbitals is typical for MR-TADF compounds (Figure 3). ${ }^{13}$ The HOMO density is mainly localized on the nitrogen and carbon atoms positioned ortho to them, while in the LUMO, electron density is mainly localized on the boron atoms and the carbons ortho to them. We applied spin component scaling second order approximate coupled-cluster (SCS-CC2) to 
accurately predict the nature and energies of the excited states and the $\Delta E_{\mathrm{ST}}$ (Figure $\mathbf{3 b}$ and Table S1). ${ }^{13,19,32} \boldsymbol{\alpha}$-3BNMes shows a gratifyingly smaller $\Delta E_{\mathrm{ST}}$ of $0.20 \mathrm{eV}$ compared to that of $\boldsymbol{\alpha}$-3BNOH $\left(\Delta E_{\mathrm{ST}}\right.$ of $\left.0.29 \mathrm{eV}\right)$, but at the expense of a slightly smaller oscillator strength $(f)$ for the transition to $\mathrm{S}_{1}$ of 0.08 compared to $\boldsymbol{\alpha - 3 B N O H ~ ( ~} f$ of 0.09 ).$^{30}$ The predicted $\mathrm{S}_{1}$ energy is also stabilized to $3.40 \mathrm{eV}$ compared to $3.69 \mathrm{eV}$ in $\boldsymbol{\alpha - 3 B N O H}$. The difference density plot of the $\mathrm{S}_{1}$ state shows the characteristic alternating pattern of increasing and decreasing electron density on adjacent atoms that is characteristic of MR-TADF compounds. ${ }^{32}$ The patterns revealed in the difference density plots for $T_{1}$ and $T_{2}$ differ slightly from that of $\mathrm{S}_{1}$. This difference in the nature of the excited singlet and triplet states will result in enhanced spin-orbit coupling and assist in RISC following El Sayed's rules. ${ }^{33}$

The absorption spectrum was simulated by capturing transitions to the first five singlet excited states at the SCS-CC2/cc-pVDZ level of theory using DFT calculated ground state. Good agreement between the measured and simulated spectral shapes was obtained (Figure S9). Similar trends in the difference density pictures of the lowest-lying singlet excitations were obtained for $\boldsymbol{\alpha}-\mathbf{3 B N M e s}$ (Figure S10) compared to those of $\boldsymbol{\alpha}-\mathbf{3 B N O H},{ }^{30}$ explaining the near identical shapes of their absorption spectra (Figure 4).

\section{Optical properties}

Steady-state photophysical properties of both $\boldsymbol{\alpha - 3 B N M e s}$ and the previous $\boldsymbol{\alpha}-\mathbf{3 B N O H}$ in dilute THF are shown in Figure 4. In the case of $\boldsymbol{\alpha}$-3BNMes, a bathochromic shift of $330 \mathrm{meV}$ compared to $\boldsymbol{\alpha}$-3BNOH is observed in both the main $\pi-\pi^{*}$ absorption band at $3.50 \mathrm{eV}(354 \mathrm{~nm})$ and the quasi degenerate $\mathrm{S}_{1}, \mathrm{~S}_{2}$ SRCT excited states at $2.94 \mathrm{eV}(421 \mathrm{~nm})$. By replacing the strongly mesomerically electron-donating hydroxyl groups with inductively electron-withdrawing mesityl substituents, both the HOMO and LUMO levels are stabilized although the LUMO to a greater extent. This results in a net stabilization of the excited states in $\boldsymbol{\alpha}$-3BNMes, and red-shifts both its absorption and emission spectra. The molar extinction coefficient for the high-energy band $\left(\mathrm{S}_{4}\right)$ is also increased by a factor of two, although this still appears at very short wavelengths, and thus is unsuitable for FRET and HF OLED applications using available D-A TADF assistant dopants. Nonetheless, this demonstrates that significant control 
over the absorption spectrum is indeed possible. Promisingly, the HF device-relevant SRCT absorption bands are increased by a factor of 1.4 along with a useful red-shift to wavelengths beyond $400 \mathrm{~nm}$ in $\alpha$ 3BNMes. Although these device-relevant SRCT bands remain smaller and at higher energies than in $\boldsymbol{v}$ DABNA (Figure S11, limiting HF compatibility with available D-A TADF co-hosts), they still represent a significant improvement compared to $\boldsymbol{\alpha - 3 B N O H}$. It remains unclear how the structure of $\boldsymbol{v}$ DABNA leads to its lowest-energy absorbance band becoming dominant, compared to the $\mathrm{S}_{4}$ band in the heptacene systems.

The solution photoluminescence spectrum of $\boldsymbol{\alpha}$-3BNMes shows a narrow emission centred at $\lambda_{\mathrm{PL}}=2.80$ eV (442 nm), with FWHM of $190 \mathrm{meV}(30 \mathrm{~nm})$ and a small Stokes shift of $140 \mathrm{meV}(20 \mathrm{~nm})$. Gratifyingly, the replacement of the hydroxyl groups with the mesityl substituents leads to a yet narrower, deep-blue emission, shifting the CIE coordinates of the PL spectrum from $(0.17,0.01)$ to $(0.15,0.04)$. These CIE 1931 values are considered ideal for blue OLEDs as defined by BT.2020-2. ${ }^{1}$ Together, these THF solution results establish the absorption- and emission-tuning abilities of the boron substituents towards synthetic control of the singlet states of the B,N-heptacene core. 


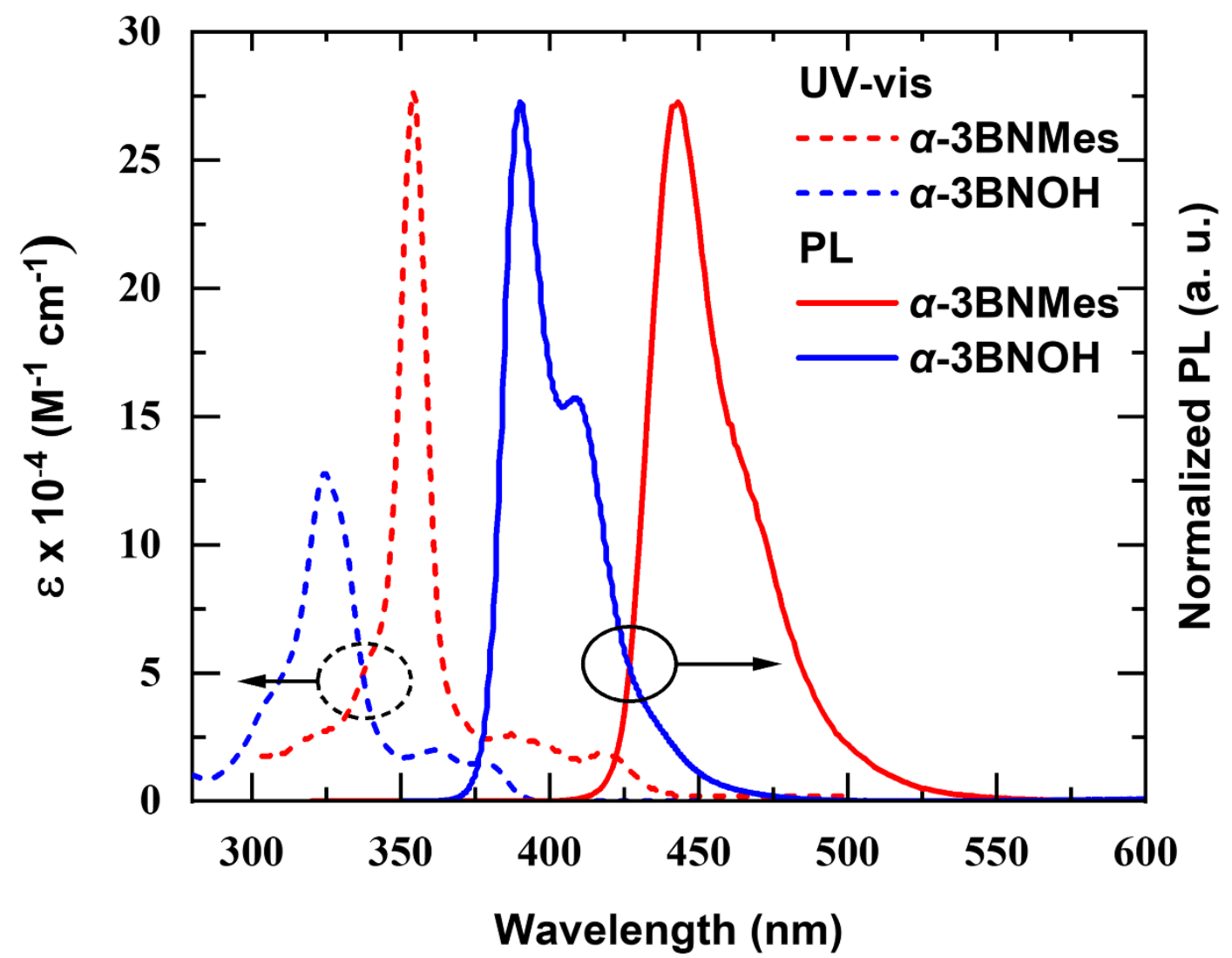

Figure 4. Absorption and emission spectra of $\alpha-3$ BNMes and $\boldsymbol{\alpha}-\mathbf{3 B N O H}$ in dilute THF solution. $\lambda_{\mathrm{exc}}=$ $310 \mathrm{~nm}$.

The solid-state fluorescence and phosphorescence spectra are shown in Figure 5a. As we have already established the red-shifting behaviour of the singlet states in identical THF environments, this allows us to consider the trends of the energies of the triplet states (from film phosphorescence) more broadly. We find that the $\Delta E_{\mathrm{ST}}$ are very similar in both materials, indicating that mesityl substitution of the boron atoms results in similar changes to both the singlet and triplet state energies of $\boldsymbol{\alpha}$-3BNMes compared to $\alpha-3 B N O H$.

Time-resolved photoluminescence decays of $\boldsymbol{\alpha}$-3BNMes doped at $1 \mathrm{wt} \%$ in PMMA matrix are shown in Figure 5b. Similar to previous reports of the photophysics of $\boldsymbol{\alpha}-\mathbf{3 B N O H} \mathbf{H}^{30}$ and many other reported MR-TADF materials, the delayed fluorescence from $\boldsymbol{\alpha}-\mathbf{3 B N M e s}$ is weak and long-lived compared to D-A TADF materials, ${ }^{8,9,34,35}$ indicating only a moderate rate of rISC. When measured at lower temperatures, the delayed emission component (after $100 \mathrm{ns)}$ is suppressed, evidencing the thermally activated 
mechanism of this emission. At longer delay times (beyond $0.1 \mathrm{~ms}$ ) and at temperatures below $150 \mathrm{~K}$ the emission decay rate changes significantly, which along with a strong spectral red-shift identifies the phosphorescence regime from which the phosphorescence spectrum (Figures 5a, 5c) is extracted.

Exponential fitting of the room temperature decay reveals three different components: the prompt fluorescence has a lifetime, $\tau_{\mathrm{p}}$, of $10 \mathrm{~ns}$, and there are two main components to the delayed emission with lifetimes of $9.08 \mu$ s and $7.06 \mathrm{~ms}$ (Figure S12a). The shorter of the delayed lifetimes, corresponding to a regime of dual emission, is attributed to combined monomer and a red-shifted aggregate emission (Figures 5c, S12c), most probably associated with excimer emission as described by Stavrou et al., ${ }^{15}$ which is common in planar MR-TADF emitters. Notably, the contribution to the emission decay from this species is minimal and energetically is at the same positions as for $\boldsymbol{\alpha - 3 B N O H} \cdot{ }^{30}$ The longest delayed component is attributed to pure monomer emission with mono-excitonic origin, in contrast with $\alpha$ 3BNOH where it was found to have bi-excitonic TTA contribution (Figure S13). As in other recent studies of MR-TADF materials, ${ }^{15,18}$ we find that changing the host matrix has only a modest influence on $k_{\text {RISC }}$ (Figure S12b). Finally, the photoluminescence quantum yield (PLQY) of $\boldsymbol{\alpha}$-3BNMes in $1 \%$ $\mathrm{mCP}$ doped film was determined to be $63 \%$, thus nearly double that of $\boldsymbol{\alpha}-\mathbf{3 B N O H}$ ( $35 \%$ in $1 \mathrm{wt} \% \mathrm{mCP}$ ), and is sufficiently high to support OLED applications. 

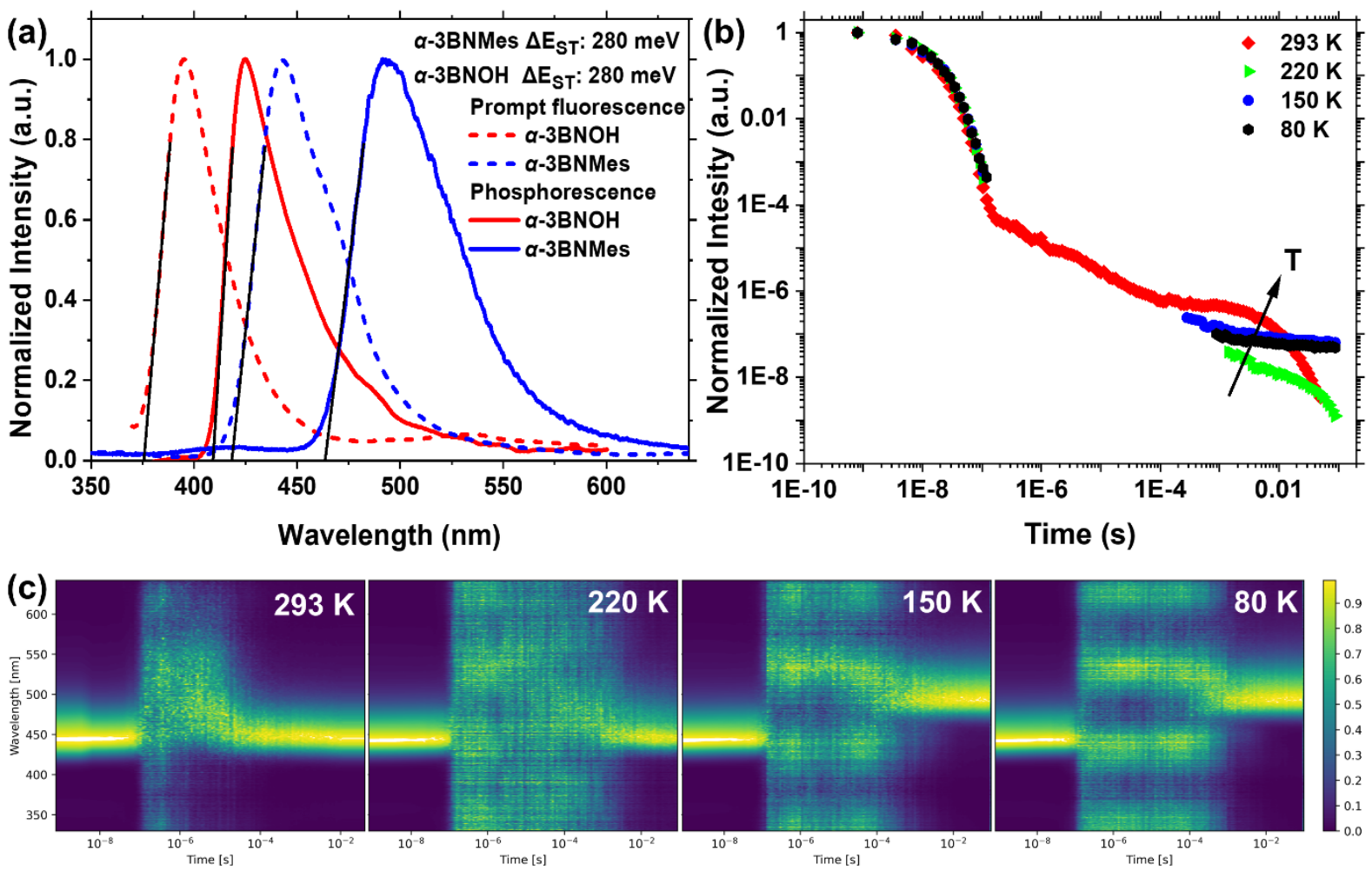

Figure 5. (a) Time-resolved prompt fluorescence (prompt, delay time $10 \mathrm{~ns}$ and gate width $5 \mathrm{~ns}$ ) and phospho-rescence (delayed, delay time $70 \mathrm{~ms}$ and gate width $15 \mathrm{~ms}$ ) spectra of $\boldsymbol{\alpha}-\mathbf{3 B N O H}{ }^{30}$ and $\boldsymbol{\alpha}$ 3BNMes $1 \mathrm{wt} \%$ in PMMA, at $80 \mathrm{~K}$. (b) Temperature-dependent time-resolved emission decay of $\boldsymbol{\alpha}$ 3BNMes $1 \mathrm{wt} \%$ in PMMA. (c) Contour plots of time-resolved emission spectra at various temperatures (regions between $100 \mathrm{~ns}$ and $100 \mu \mathrm{s}$ at lower temperatures represent background collection, i.e., no emis-sion). $\lambda_{\text {exc }}=355 \mathrm{~nm}$.

\section{Devices}

Having confirmed the weak TADF activity of $\boldsymbol{\alpha - 3 B N M e s , ~ i t s ~ u s e ~ a s ~ a n ~ e m i t t e r ~ i n ~ O L E D s ~ w a s ~ n o n e t h e - ~}$ less preliminarily assessed. Devices using a stack of ITO (anode) $\mid$ NPB (HIL/HTL, $40 \mathrm{~nm}$ ) $\mid \mathrm{mCBP}$ (EBL, $10 \mathrm{~nm}) \mid \boldsymbol{\alpha}$-3BNMes:host X\% (EML, $30 \mathrm{~nm}) \mid$ T2T (HBL, $10 \mathrm{~nm}$ ) | T2T:LiQ 45\% (EIL/ETL, 35 $\mathrm{nm}) \mid \mathrm{Al}$ (cathode, $100 \mathrm{~nm}$ ) were fabricated, with representative performance shown in Figure S14. Two different hosts were used, DPEPO (electron transporting, $\mathrm{E}_{\mathrm{T}}=3.05 \mathrm{eV}$ ) and $\mathrm{mCBP}$ (hole transporting and otherwise similar to $\mathrm{mCP}$, and with $\mathrm{E}_{\mathrm{T}}=2.85 \mathrm{eV}$ now confirmed high enough to be compatible with the emitter $\mathrm{E}_{\mathrm{T}}=2.7 \mathrm{eV}$ ), with varying concentrations of $\boldsymbol{\alpha}$-3BNMes ranging from $5 \mathrm{vol} \%$ up to $20 \mathrm{vol} \%$. With increasing doping concentration, no significant differences were observed in the current densityvoltage-luminance (jVL) curvers, the spectra, or EQE as a function of current density in both host environments. Consequently, we do not anticipate qualitatively different behaviour in MR-TADF only 
devices with lower doping concentration, which were thus not investigated. More interestingly, no broadening of the electroluminescence (EL) spectrum was observed upon increasing concentration, indicating suppression of excimer formation even at high concentrations, a very common phenomenon of other MR-TADF emitters in films. Maximum EQEs of $1.7 \%$ at $\sim 100 \mathrm{~cd} \mathrm{~m}^{-2}$ were achieved in devices with each of the two hosts. We suggest that this poor performance is due to the weak TADF contribution of the material, leading to low efficiency combined with additional efficiency roll-off from the known instability of DPEPO, ${ }^{36,37}$ especially at high current densities (Figure S14c). Nonetheless, the CIE coordinates are very attractive at $(0.15,0.08)$, which are the same as in the photoexcited film and similar to that determined in THF solution.

To compensate for the poor intrinsic exciton harvesting performance of $\boldsymbol{\alpha}$-3BNMes while still taking advantage of its ideal emission spectrum we then applied it as a terminal emitter in HF OLEDs in combination with a D-A(-D) TADF sensitizer. In order to ensure adequate spectral overlap necessary for the energy transfer, we employed 2,8-bis(2,7-di(tert-butyl)-9,9-dimethylacridin-10(9H)yl)dibenzo[b,d]thiophene (DtBuAc-DBT) as a TADF co-host, previously reported to give $\sim 11 \%$ EQEmax and suitably high-energy blue emission with CIE coordinates of $(0.16,0.17) .{ }^{8}$ This D-A-D TADF assistant dopant was co-evaporated at $25 \mathrm{vol} \%$ in the EML, alongside $1 \mathrm{vol} \% \boldsymbol{\alpha}$-3BNMes and $74 \mathrm{vol} \%$ DPEPO. The device architecture was chosen to be the same as the one previously reported, ${ }^{8}$ with the addition of $\boldsymbol{a}$-3MesBN as the terminal emitter, using ITO (anode) $\mid$ NPB (HTL, $40 \mathrm{~nm}$ ) | TSBPA (EBL, $10 \mathrm{~nm}$ ) $\boldsymbol{\alpha}$-3BNMes:DtBuAc-DBT:DPEPO 1:25:74\% (EML, $30 \mathrm{~nm}$ ) | DPEPO (HBL, $10 \mathrm{~nm}$ ) | TBPi (ETL, $40 \mathrm{~nm})|\mathrm{LiF}(\mathrm{EIL}, 1 \mathrm{~nm})| \mathrm{Al}$ (cathode, $100 \mathrm{~nm}$ ). Both control "D-A-D only" (TADF) and combined HF OLED devices were produced in the same deposition run to provide a suitable comparison. 
(a)

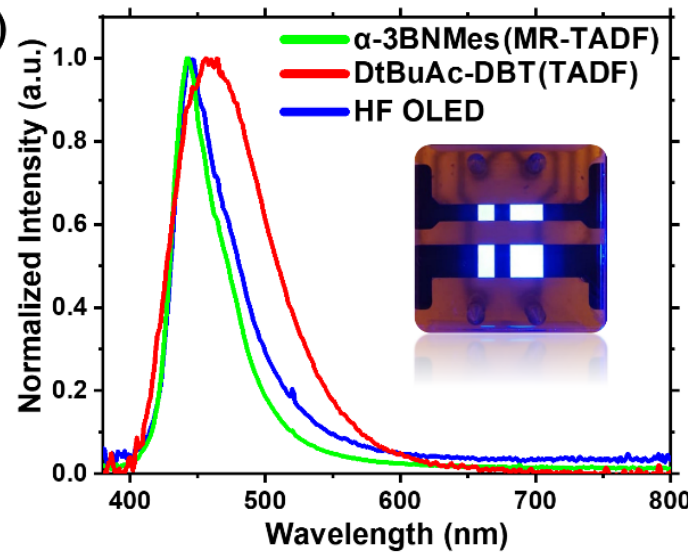

(c)

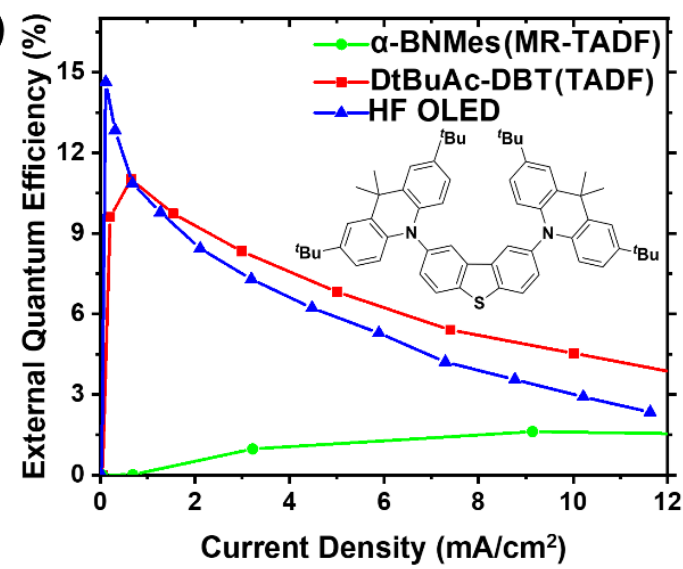

(b)

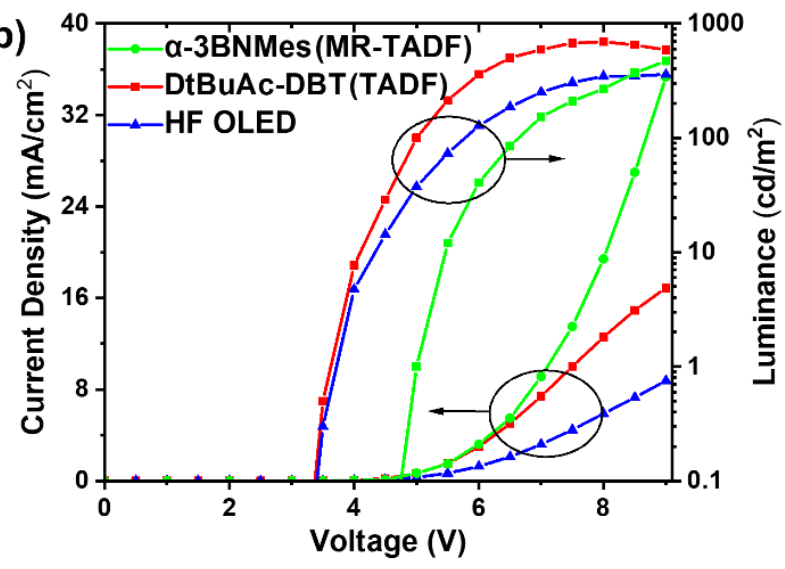

(d)

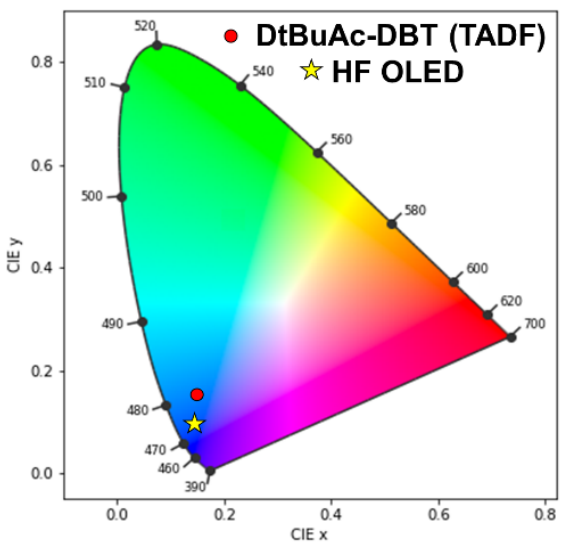

Figure 6. Performance of MR-TADF (green), D-A-D (red), and HF (blue) OLEDs. (a) EL spectra. Inset: Operating HF OLED. (b) jVL characteristics. (c) EQE vs current density. Inset: DtBuAc-DBT structure. (d) CIE coordinates.

The resulting HF OLEDs possess good efficiency, with an $\mathrm{EQE}_{\max }$ of $15 \%$ and an $\mathrm{EQE}_{100}\left(\right.$ at $100 \mathrm{~cd} / \mathrm{m}^{2}$ ) of $10.2 \%$, with a FWHM of $290 \mathrm{meV}(49 \mathrm{~nm})$ and CIE of $(0.15,0.10)$, which is enabled by triplet harvesting by the D-A-D sensitizer, together with narrow deep blue emission from the $\boldsymbol{\alpha}$-3BNMes (Figure 6). Although the $\mathrm{EQE}_{\max }$ is improved at $15 \%$ in the HF OLED (compared to $11 \%$ for the DtBuAcDBT device) the larger roll-off at higher current densities results to an $\mathrm{EQE}_{300}$ of $6.2 \%$ and $8.8 \%$ for the HF and TADF OLEDs, respectively. The increase in $\mathrm{EQE}_{\max }$ is a compromise between detrimental factors (e.g., the moderate PLQY of $\boldsymbol{\alpha}$-3BNMes as the terminal emitter, competing unproductive Dexter energy transfer, in-situ charge trapping, etc.) and beneficial effects (e.g., $\boldsymbol{\alpha}$-3BNMes alignment/anisotropic emission, and good FRET overlap outcompeting DtBuAc-DBT non-radiative decay), although some of these factors are deceptively difficult to quantify by established experimental methods. ${ }^{25}$ The 
overall HF OLED performance is nonetheless competitive with other recent leading reports at similar colour coordinates (Figure S16 and Table S2).

The EL spectrum of the HF OLEDs is nearly identical to the $\boldsymbol{\alpha}$-3BNMes spectrum, indicating efficient FRET with only a small contribution of the D-A-D TADF sensitizer at $\sim 525 \mathrm{~nm}$. This efficient FRET occurs despite a seemingly small FRET overlap (Figure S15) and highlights the importance and challenge of both emission and absorption spectral tuning for developing high-efficiency blue HF OLED material combinations. While it is incredibly challenging to further deepen the emission colour of available D-A-D TADF assistant dopants, the need to do so can be avoided in HF OLEDs by instead redshifting the absorption spectrum of the MR-TADF terminal emitters, as we demonstrate here.

\section{Conclusions}

We have achieved emission and absorption colour tuning in a deep blue non-triangulene type MRTADF compound by altering the boron substituents in a B, N-doped heptacene. Compared to the parent UV emitter $\boldsymbol{\alpha}-\mathbf{3 B N O H}$, these changes imbue $\boldsymbol{\alpha}$-3BNMes with ideal CIE coordinates for blue OLEDs and suitable absorption spectrum for FRET compatibility with existing D-A TADF co-hosts. The resulting HF OLEDs achieved an $\mathrm{EQE}_{\max }$ of $15 \%$ and deep-blue colour coordinates of $(0.15,0.10)$, compared to $<1 \%$ efficiencies for the unassisted $\boldsymbol{\alpha - 3 B N O H}$. In light of slow progress towards the development of truly deep-blue D-A TADF emitters, we advance that controlling the absorption spectrum of terminal MR-TADF emitters to expand their compatibility in HF devices is a more fruitful approach.

\section{Supporting Information}

${ }^{1}$ HNMR, HRMS and HPLC trace of $\boldsymbol{\alpha}$-3BNMes; TGA curve; supplementary computational data and coordinates; Crystallographic data for $\boldsymbol{\alpha}$-3BNMes (CIF). Additional photophysical and OLED data.

\section{Acknowledgments}


This project has received funding from the European Union's Horizon 2020 research and innovation programme under the Marie Skłodowska Curie grant agreement No 838885 (NarrowbandSSL) and under the Marie Skłodowska Curie grant agreement No 812872 (TADFlife). S.M.S. acknowledges support from the Marie Skłodowska-Curie Individual Fellowship (grant agreement No 838885 NarrowbandSSL). The St Andrews team would like to thank the Leverhulme Trust (RPG-2016-047) for financial support. E. Z.-C. is a Royal Society Leverhulme Trust Senior Research fellow (SRF\R1\201089). Computational resources have been provided by the Consortium des Équipements de Calcul Intensif (CÉCI), funded by the Fonds de la Recherche Scientifiques de Belgique (F. R. S.-FNRS) under Grant No. 2.5020.11, as well as the Tier-1 supercomputer of the Fédération Wallonie-Bruxelles, infrastructure funded by the Walloon Region under the grant agreement n1117545. The authors thank F. Rodella and Prof. P. Strohriegl from Bayreuth University for help with the thermogravimetric analysis.

\section{References}

(1) ITU. Recommendation ITU-R BT.2020-2: Parameter Values for Ultra-High Definition Television Systems for Production and International Programme Exchange. Recommendation ITU-R BT.2020. 2015.

(2) Seidel, R. Introduction to the SMPTE 2014 Progress Report. SMPTE Motion Imaging Journal. 2014. https://doi.org/10.5594/j18442.

(3) Takita, Y.; Takeda, K.; Hashimoto, N.; Nomura, S.; Suzuki, T.; Nakashima, H.; Uesaka, S.; Seo, S.; Yamazaki, S. Highly Efficient Deep-Blue Fluorescent Dopant for Achieving LowPower OLED Display Satisfying BT.2020 Chromaticity. J. Soc. Inf. Disp. 2018. https://doi.org/10.1002/jsid.634.

(4) Hashimoto, N.; Takita, Y.; Yamaoka, R.; Nomura, S.; Sasaki, T.; Yamagata, S.; Suzuki, T.; Seo, S. 52-4: Long-Lived Thermally Stable Blue OLED Achieving BT.2020 Color Gamut. SID Symp. Dig. Tech. Pap. 2017. https://doi.org/10.1002/sdtp.11750.

(5) Wong, M. Y.; Zysman-Colman, E. Purely Organic Thermally Activated Delayed Fluorescence Materials for Organic Light-Emitting Diodes. Advanced Materials. 2017. 
https://doi.org/10.1002/adma.201605444.

(6) Ansari, R.; Shao, W.; Yoon, S. J.; Kim, J.; Kieffer, J. Charge Transfer as the Key Parameter Affecting the Color Purity of Thermally Activated Delayed Fluorescence Emitters. ACS Appl. Mater. Interfaces 2021. https://doi.org/10.1021/acsami.1c02943.

(7) Stavrou, K.; Franca, L. G.; Monkman, A. P. Photophysics of TADF Guest - Host Systems: Introducing the Idea of Hosting Potential. 2020. https://doi.org/10.1021/acsaelm.0c00514.

(8) Huang, R.; Kukhta, N. A.; Ward, J. S.; Danos, A.; Batsanov, A. S.; Bryce, M. R.; Dias, F. B. Balancing Charge-Transfer Strength and Triplet States for Deep-Blue Thermally Activated Delayed Fluorescence with an Unconventional Electron Rich Dibenzothiophene Acceptor. $J$. Mater. Chem. C 2019. https://doi.org/10.1039/c9tc02175b.

(9) Stachelek, P.; Ward, J. S.; Dos Santos, P. L.; Danos, A.; Colella, M.; Haase, N.; Raynes, S. J.; Batsanov, A. S.; Bryce, M. R.; Monkman, A. P. Molecular Design Strategies for Color Tuning of Blue TADF Emitters. ACS Appl. Mater. Interfaces 2019, 11 (30), 27125-27133. https://doi.org/10.1021/acsami.9b06364.

(10) Dos Santos, P. L.; Chen, D.; Rajamalli, P.; Matulaitis, T.; Cordes, D. B.; Slawin, A. M. Z.; Jacquemin, D.; Zysman-Colman, E.; Samuel, I. D. W. Use of Pyrimidine and Pyrazine Bridges as a Design Strategy to Improve the Performance of Thermally Activated Delayed Fluorescence Organic Light Emitting Diodes. ACS Appl. Mater. Interfaces 2019. https://doi.org/10.1021/acsami.9b16952.

(11) Wright, I. A.; Danos, A.; Montanaro, S.; Batsanov, A. S.; Monkman, A. P.; Bryce, M. R. Conformational Dependence of Triplet Energies in Rotationally Hindered N- and SHeterocyclic Dimers: New Design and Measurement Rules for High Triplet Energy OLED Host Materials. Chem. - A Eur. J. 2021. https://doi.org/10.1002/chem.202100036.

(12) Ha, J. M.; Hur, S. H.; Pathak, A.; Jeong, J. E.; Woo, H. Y. Recent Advances in Organic Luminescent Materials with Narrowband Emission. NPG Asia Materials. 2021. 
https://doi.org/10.1038/s41427-021-00318-8.

(13) Madayanad Suresh, S.; Hall, D.; Beljonne, D.; Olivier, Y.; Zysman-Colman, E. Multiresonant Thermally Activated Delayed Fluorescence Emitters Based on Heteroatom-Doped Nanographenes: Recent Advances and Prospects for Organic Light-Emitting Diodes. Advanced Functional Materials. 2020. https://doi.org/10.1002/adfm.201908677.

(14) Monkman, A. Why Do We Still Need a Stable Long Lifetime Deep Blue OLED Emitter? ACS Applied Materials and Interfaces. 2021. https://doi.org/10.1021/acsami.1c09189.

(15) Stavrou, K.; Danos, A.; Hama, T.; Hatakeyama, T.; Monkman, A. Hot Vibrational States in a High-Performance Multiple Resonance Emitter and the Effect of Excimer Quenching on Organic Light-Emitting Diodes. ACS Appl. Mater. Interfaces 2021. https://doi.org/10.1021/acsami.0c20619.

(16) Northey, T.; Penfold, T. J. The Intersystem Crossing Mechanism of an Ultrapure Blue Organoboron Emitter. Org. Electron. 2018. https://doi.org/10.1016/j.orgel.2018.04.038.

(17) Kim, I.; Cho, K. H.; Jeon, S. O.; Son, W.-J.; Kim, D.; Rhee, Y. M.; Jang, I.; Choi, H.; Kim, D. S. Three States Involving Vibronic Resonance Is a Key to Enhancing Reverse Intersystem Crossing Dynamics of an Organoboron-Based Ultrapure Blue Emitter. JACS Au 2021. https://doi.org/10.1021/jacsau.1c00179.

(18) Hall, D.; Stavrou, K.; Duda, E.; Danos, A.; Bagnich, S.; Warriner, S.; Slawin, A. M. Z.; Beljonne, D.; Köhler, A.; Monkman, A.; Olivier, Y.; Zysman-Colman, E. Diindolocarbazole Achieving Multiresonant Thermally Activated Delayed Fluorescence without the Need for Acceptor Units. Mater. Horizons 2022. https://doi.org/10.1039/d1mh01383a.

(19) Pershin, A.; Hall, D.; Lemaur, V.; Sancho-Garcia, J. C.; Muccioli, L.; Zysman-Colman, E.; Beljonne, D.; Olivier, Y. Highly Emissive Excitons with Reduced Exchange Energy in Thermally Activated Delayed Fluorescent Molecules. Nat. Commun. 2019, 10 (1), 597. https://doi.org/10.1038/s41467-019-08495-5. 
(20) Hall, D.; Suresh, S. M.; dos Santos, P. L.; Duda, E.; Bagnich, S.; Pershin, A.; Rajamalli, P.; Cordes, D. B.; Slawin, A. M. Z.; Beljonne, D.; Köhler, A.; Samuel, I. D. W.; Olivier, Y.; Zysman-Colman, E. Improving Processability and Efficiency of Resonant TADF Emitters: A Design Strategy. Adv. Opt. Mater. 2020, 8 (2), 1901627. https://doi.org/10.1002/adom.201901627.

(21) Zysman-Colman, E. Molecular Designs Offer Fast Exciton Conversion. Nature Photonics. 2020. https://doi.org/10.1038/s41566-020-0696-8.

(22) Jeon, S. O.; Lee, K. H.; Kim, J. S.; Ihn, S. G.; Chung, Y. S.; Kim, J. W.; Lee, H.; Kim, S.; Choi, H.; Lee, J. Y. High-Efficiency, Long-Lifetime Deep-Blue Organic Light-Emitting Diodes. Nat. Photonics 2021. https://doi.org/10.1038/s41566-021-00763-5.

(23) Zhang, D.; Song, X.; Gillett, A. J.; Drummond, B. H.; Jones, S. T. E.; Li, G.; He, H.; Cai, M.; Credgington, D.; Duan, L. Efficient and Stable Deep-Blue Fluorescent Organic Light-Emitting Diodes Employing a Sensitizer with Fast Triplet Upconversion. Adv. Mater. 2020. https://doi.org/10.1002/adma.201908355.

(24) Chan, C.-Y.; Tanaka, M.; Lee, Y.-T.; Wong, Y.-W.; Nakanotani, H.; Hatakeyama, T.; Adachi, C. Stable Pure-Blue Hyperfluorescence Organic Light-Emitting Diodes with High-Efficiency and Narrow Emission. Nat. Photonics 2021. https://doi.org/10.1038/s41566-020-00745-z.

(25) Haase, N.; Danos, A.; Pflumm, C.; Stachelek, P.; Brütting, W.; Monkman, A. P. Are the Rates of Dexter Transfer in TADF Hyperfluorescence Systems Optically Accessible? Mater. Horizons 2021. https://doi.org/10.1039/d0mh01666g.

(26) Nakanotani, H.; Higuchi, T.; Furukawa, T.; Masui, K.; Morimoto, K.; Numata, M.; Tanaka, H.; Sagara, Y.; Yasuda, T.; Adachi, C. High-Efficiency Organic Light-Emitting Diodes with Fluorescent Emitters. Nat. Commun. 2014. https://doi.org/10.1038/ncomms5016.

(27) Tanaka, H.; Oda, S.; Ricci, G.; Gotoh, H.; Tabata, K.; Kawasumi, R.; Beljonne, D.; Olivier, Y.; Hatakeyama, T. Hypsochromic Shift of Multiple-Resonance-Induced Thermally Activated 
Delayed Fluorescence by Oxygen Atom Incorporation. Angew. Chemie - Int. Ed. 2021. https://doi.org/10.1002/anie.202105032.

(28) Yang, M.; Park, I. S.; Yasuda, T. Full-Color, Narrowband, and High-Efficiency Electroluminescence from Boron and Carbazole Embedded Polycyclic Heteroaromatics. $J$. Am. Chem. Soc. 2020. https://doi.org/10.1021/jacs.0c10081.

(29) Li, J.; Zhao, F.; Chen, yanan; Zhang, M.; Li, T.; Zhang, H. Color Tuning of Di-Boron Derived TADF Emitters: Molecular Design and Property Prediction. J. Mater. Chem. C 2021. https://doi.org/10.1039/d1tc03345j.

(30) Suresh, S. M.; Duda, E.; Hall, D.; Yao, Z.; Bagnich, S.; Slawin, A. M. Z.; Bässler, H.; Beljonne, D.; Buck, M.; Olivier, Y.; Köhler, A.; Zysman-Colman, E. A Deep Blue B,N-Doped Heptacene Emitter That Shows Both Thermally Activated Delayed Fluorescence and Delayed Fluorescence by Triplet-Triplet Annihilation. J. Am. Chem. Soc. 2020, 142 (14), 6588-6599. https://doi.org/10.1021/jacs.9b13704.

(31) Suresh, S. M.; Duda, E.; Kahle, F. J.; Hall, D.; Bagnich, S.; Bässler, H.; Beljonne, D.; Olivier, Y.; Köhler, A.; Zysman-Colman, E. Design of Multi-Resonance Thermally Activated Delayed Fluorescence Materials for Organic Light-Emitting Diodes. In Digest of Technical Papers SID International Symposium; 2021. https://doi.org/10.1002/sdtp.14654.

(32) David Hall, Juan Carlos Sancho-Garcia, Anton Pershin, David Beljonne, Eli ZysmanColmana, Y. O. The Modelling of Multi-Resonant Thermally Activated Delayed Fluorescence Emitters - Properly Accounting for Electron Correlation Is Key! ChemRxiv. Prepr. 2021, 10.33774/chemrxiv-2021-496gn.

(33) Etherington, M. K.; Gibson, J.; Higginbotham, H. F.; Penfold, T. J.; Monkman, A. P. Revealing the Spin-Vibronic Coupling Mechanism of Thermally Activated Delayed Fluorescence. Nat. Commun. 2016, 7 (1), 13680. https://doi.org/10.1038/ncomms13680.

(34) Ward, J. S.; Danos, A.; Stachelek, P.; Fox, M. A.; Batsanov, A. S.; Monkman, A. P.; Bryce, 
M. R. Exploiting Trifluoromethyl Substituents for Tuning Orbital Character of Singlet and Triplet States to Increase the Rate of Thermally Activated Delayed Fluorescence. Mater. Chem. Front. 2020. https://doi.org/10.1039/d0qm00429d.

(35) Kukhta, N. A.; Higginbotham, H. F.; Matulaitis, T.; Danos, A.; Bismillah, A. N.; Haase, N.; Etherington, M. K.; Yufit, D. S.; McGonigal, P. R.; Gražulevičius, J. V.; Monkman, A. P. Revealing Resonance Effects and Intramolecular Dipole Interactions in the Positional Isomers of Benzonitrile-Core Thermally Activated Delayed Fluorescence Materials. J. Mater. Chem. C 2019. https://doi.org/10.1039/c9tc02742d.

(36) Ihn, S. G.; Lee, N.; Jeon, S. O.; Sim, M.; Kang, H.; Jung, Y.; Huh, D. H.; Son, Y. M.; Lee, S. Y.; Numata, M.; Miyazaki, H.; Gómez-Bombarelli, R.; Aguilera-Iparraguirre, J.; Hirzel, T.; Aspuru-Guzik, A.; Kim, S.; Lee, S. An Alternative Host Material for Long-Lifespan Blue Organic Light-Emitting Diodes Using Thermally Activated Delayed Fluorescence. Adv. Sci. 2017. https://doi.org/10.1002/advs.201600502.

(37) Ihn, S.; Jeong, D.; Kwon, E. S.; Kim, S.; Chung, Y. S.; Sim, M.; Chwae, J.; Koishikawa, Y.; Jeon, S. O.; Kim, J. S.; Kim, J.; Nam, S.; Kim, I.; Park, S.; Kim, D. S.; Choi, H.; Kim, S. Dipole Moment- and Molecular Orbital-Engineered Phosphine Oxide-Free Host Materials for Efficient and Stable Blue Thermally Activated Delayed Fluorescence. Adv. Sci. 2021, 2102141, 2102141. https://doi.org/10.1002/advs.202102141.

TOC 


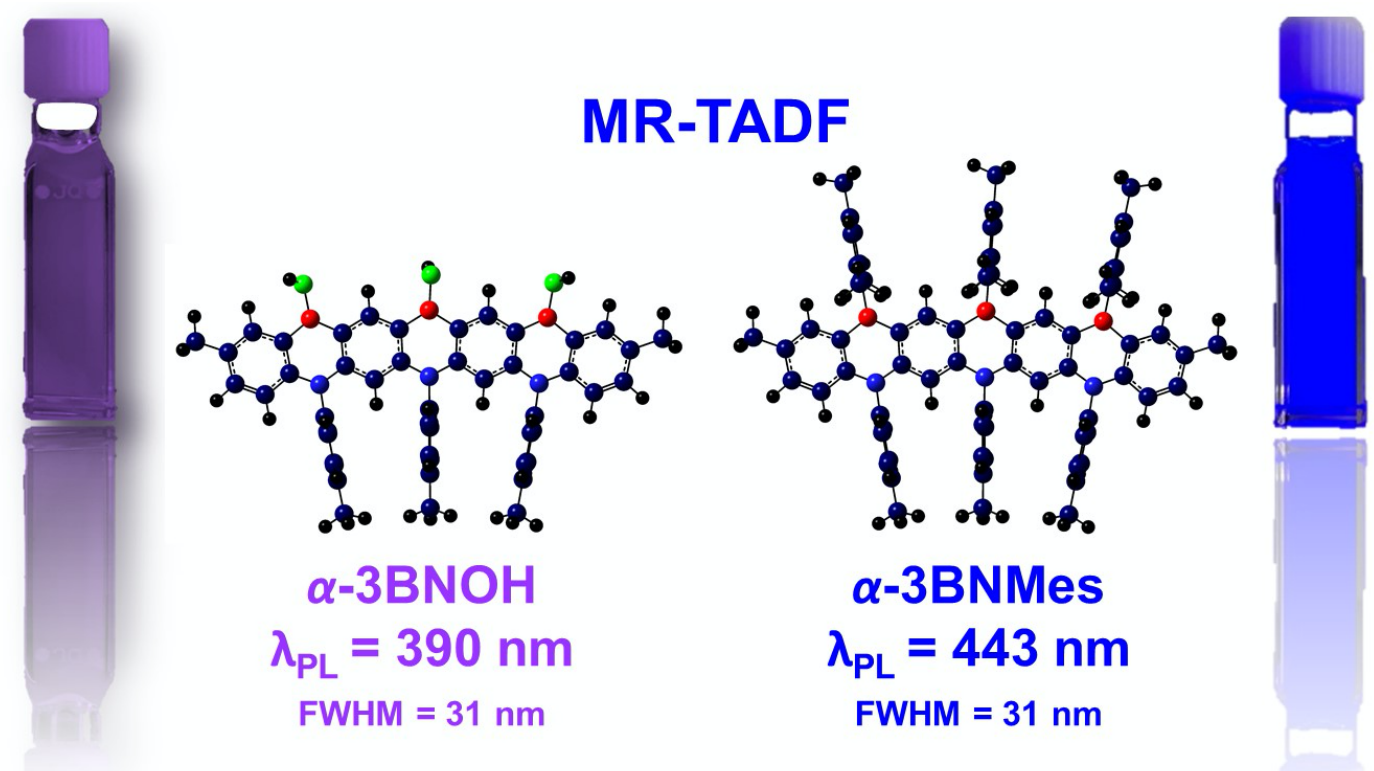

